\title{
Thymidine Kinase 1: Making Its Mark In Immunotherapy
}

\author{
Toni O. Mortimer, Rachel Morris, Abigail Schekall, Kai Barlow, Kim L. O’Neill
}

Department of Microbiology and Molecular Biology, Brigham Young University, Provo, Utah 84602

\section{Article Info}

\section{Article Notes}

Received: November 09, 2021

Accepted: December 08, 2021

\section{*Correspondence:}

${ }^{*}$ Dr. Kim L. O'Neill, Department of Microbiology and Molecular Biology, Brigham Young University, Provo, Utah 84602; Email: kim_oneill@byu.edu.

${ }^{\circledR} 2021$ O'Neill KL. This article is distributed under the terms of the Creative Commons Attribution 4.0 International License.

\section{Keywords:}

Thymidine kinase 1

CAR-T cell therapy

Biomarker

Immunotherapy

Melanoma

Salvage pathway

Monoclonal antibody

\section{Abstract}

Cancer is one of the leading causes of death worldwide. In the U.S. alone, almost 2 million people will be diagnosed with cancer each year and just over a quarter of those diagnosed will pass away from the disease. Skin cancers are the most common forms of cancer. Early detection of cancer and cancer biomarkers enables clearer understanding of cancer progression in a patient and more effective treatments in response to the disease. Clinically relevant biomarkers are not only tools for early diagnosis of cancer but may also prove useful as immune targets for various immunotherapies, such as monoclonal antibody-based therapy and chimeric antigen receptor (CAR) T-cell therapy. This review provides a brief overview of the rescue pathway enzyme thymidine kinase 1 (TK1) and its history and biology, as well as discusses its role as a biomarker and potential immune target.

\section{Introduction}

Cancer biomarkers are crucial for diagnosis and prognosis. One protein widely recognized as a clinically valuable biomarker and potential immune target is salvage pathway enzyme thymidine kinase 1 (TK1). With the discovery of DNA structure and replication methods in the 1950s, research since then has produced significant discoveries regarding TK1, its characterization and functions. The regeneration of thymidine for DNA synthesis and damage is accomplished in part by TK1 ${ }^{1}$. In 1983 , Bradshaw et al. was able to sequence the TK1 gene and identify a section within the $5^{\prime}$ end region that induced S phase activity ${ }^{2}$. TK1 upregulation in the $S$ phase of the cell cycle allows the enzyme to become an indicator for active cell proliferation $^{3-5}$. Because of its upregulation in various cancers, TK1 is a significant biomarker for cancer and cancer stage ${ }^{4}$. While TK1 is known to be involved in cancer progression, there is limited research specifically focused on the correlations between TK1 and the progression of skin cancers. One study does emphasize a correlation between serum TK1 and metastatic sites in melanoma, indicating that the enzyme may be involved in the progression of melanoma metastasis ${ }^{6}$. This mini review expands on a general history of TK1 and concludes with an exploration of recent discoveries and further applications for TK1 with regard to novel immunotherapies.

\section{Role of TK1 as a Salvage Pathway Enzyme}

TK1 is a DNA salvage pathway enzyme that is responsible for recycling and regenerating thymidine for DNA synthesis. Generally, the de novo pathway is also utilized by cells for regenerating nucleotides; however, the salvage pathway is less energy expensive. Thymidine crosses the cell membrane and enters the cytosol via facilitated diffusion and is then phosphorylated into its 
monophosphate form (dTMP) by TK17. Following this first step, other enzymes are recruited to convert dTMP to deoxythymidine triphosphate (dTTP), its triphosphate form. Nucleotides such as dTTP enter the nucleus via passive transport ${ }^{8}$. In addition to DNA synthesis, TK1 plays an important role in managing DNA repair because it generates a supply of dTTP that is readily available to replace damaged nucleotides. DNA damage caused by radiation or chemotherapy often results in increased TK1 levels ${ }^{1}$. TK1 is essential to repair DNA damage, and required to form nucleotides destined for cellular repair outside of S phase $\mathrm{e}^{1,9}$. In fact, TK1 depletion in cells with DNA damage often results in cell death ${ }^{4}$.

\section{Regulation of TK1}

TK1 plays a critical role in cell cycle regulation. Protein dysregulation and mutations may promote the breaking of cell cycle control and induce tumorigenesis ${ }^{10}$. Adler et al., performed some early studies indicating that TK1 cytosolic levels peak during $\mathrm{S}$ phase $\mathrm{e}^{11}$. During periods of DNA damage and normal cell function, mRNA levels of TK1 are controlled by the RB/E2F pathway, which is known to regulate transcription at the promoter site ${ }^{12}$. Bradshaw et al. discovered a 16-kilobase region, located on the 5 ' end of the TK1 gene, responsible for TK1 activity during S phase ${ }^{2}$. Additionally, there are important regulatory elements located in the TK1 gene's coding region and a 70 bp region within the promoter that controls TK1 expression ${ }^{13}$. Also, TK1 normally exists as a quiescent dimer and as a tetramer when active during $\mathrm{S}$ phase ${ }^{14}$. TK1 tetramer activity is allosterically controlled by dTTP levels. When dTTP binding occurs, the binding site for ATP and thymidine on TK1 is blocked, which prevents thymidine phosphorylation ${ }^{15}$. However, the phosphorylation of serine residues during post-translational modification induces TK1 activation ${ }^{14}$. Overall, TK1 has proven to be an important cell cycle protein that will continue to be researched to better elucidate its many roles.

\section{TK1 As a Biomarker for Cancer}

The discovery of TK1 was introduced in the $1960 \mathrm{~s}^{16}$ Since then, elevated levels of TK1 have been shown in various studies that focused on the sera of many different cancer types, including lung, colon, breast, and prostate ${ }^{1}$. In one study done by Alegre et al., they showed that TK1 levels were elevated in lung cancer. Their results reflected that TK1 was significantly elevated in all lung cancer samples. Patients with stage I $(n=16)$ and stage II $(n=17)$ cancer showed higher TK1 levels compared to their controls ${ }^{17}$. A study done on serum TK1 (sTK1) levels during neoadjuvant chemotherapy (NACT) for early breast cancer (BC) longitudinally assessed TK1 as a reputed long-term biomarker in $\mathrm{BC}$, both at baseline and following short-term exposure to NACT. Their results showed that
TK1 levels increased compared to baseline levels 48 hours after administration of the chemotherapy ${ }^{18}$. A clinical test done by Larsson et al. evaluated STK1 in metastatic breast cancer (MBC) patients as a tool for disease prediction and treatment monitoring. They monitored 142 women with MBC and found that higher sTK1 levels correlated with worse progression-free (PFS) and overall survival (OS) at baseline levels. During 1st line systemic therapy (ET, ChT or HER2 targeted therapy), they noticed that changes within 3 to 6 months significantly correlated with PFS and $\mathrm{OS}^{19}$. Along with this finding, a recent discovery by Liu et al. showed that the knock down of TK1 in cells reduces proliferation, invasion, and cell migration. They studied these effects on thyroid carcinoma tissues and developed strong evidence between TK1 levels and cancer prognosis ${ }^{20}$.

In a study done by Weagel et al., TK1 is confirmed to reside as monomeric and dimer forms that function on the cell membrane. They confirm that TK1 is upregulated and contained on the plasma membrane of Burkitt's lymphoma, acute promyelocytic leukemia, $\mathrm{T}$ cell leukemia, and acute lymphoblasticleukemia. This starkly contrasts their findings on normal resting or proliferating lymphocytes where TK1 upregulation does not occur ${ }^{21}$. As elevated expression of TK1 has been shown to be present in multiple cancer types, cancer development, and immune-suppression, TK1 can be developed as a beneficial tool as a tumor biomarker when testing for cancer and disease management. Its potential as a biomarker also leads to possibilities for the targeting of TK1 with immunotherapeutic treatments.

\section{Additional Factors in Immune-Targeting and Immunotherapy Administration}

In conjunction with the study of cancer biomarkers and other biological mechanisms to prevent, diagnose, and treat cancer, it is important to understand pathogenic interactions among environment, host, and tumor. These interactions lend to the understanding of the molecular pathology and epidemiology of cancer and aid in determining how a patient will respond to immunotherapy.

A major barrier for immunotherapies is the tumor microenvironment (TME). The TME is made up of cancer cells, extracellular matrix components, secreted chemokines and cytokines, signaling molecules, and infiltrating immune cells, and is involved in immune cell recruitment and activation, drug resistance, and tumor progression $^{22,23}$. The TME influences immunosuppression through the secretion of chemokines and cytokines by tumor-associated macrophages, regulatory $\mathrm{T}$ cells (Tregs), fibroblasts, and other tumor cells ${ }^{24,25}$. Rapidly proliferating tumor cells increase their nutrient uptake, produce inhibitory metabolites, and convert to an acidic environment through the production of lactic acid and carbonic acid during aerobic glycolysis and oxidative 
phosphorylation ${ }^{25,26}$. The metabolically hostile TME forces immune cells to compete for nutrients and makes it difficult for cells to survive in their hypoxic and acidic surroundings ${ }^{24,26,27}$. This interactive biological ecosystem is also affected by aging, diet, drug and alcohol consumption, adiposity, diabetes, physical exercise, and other genetic and epidemiological factors ${ }^{28}$. Navigation of the TME is crucial for adequate administration of immunotherapies.

In conjunction with the TME, continuous interaction with the immune system causes the human microbiome to also have a role in immunotherapy efficacy. Monitoring and recognition of microbes is largely performed by the innate immune system, thus, microbes and immune cell populations can be found in close proximity ${ }^{29}$. Evidence indicates that variation in the human microbiome can have carcinogenic effects ${ }^{28,30}$. These microflora alterations may include biochemical changes. One such change is the hypothesized metabolism of Thymidine Kinase by gut microflora. Data from one study shows that Thymidine Kinase (TK) activity is reduced by $50 \%$ in normal mice compared to germ free ${ }^{31}$. TK activity is known to be involved in cancer development, thus, through interaction with TK and other possible enzymes involved in cancer progression, microflora is suggested to be involved in tumor progression ${ }^{30}$. Evidence suggests that tumor phenotype may also be partially determined by specific microorganisms present ${ }^{28,32}$. Similar to the TME, human microbiota are influenced by diet, alcohol use, medications, and other lifestyle factors ${ }^{33}$.

The TME and human microbiome represent various factors involved in carcinogenesis and characterize the influence that interactions between environment, host, and tumor have on cancer progression. Continued research of these interactions is essential for effective recognition of cancer biomarkers and biomarker targeting in immunotherapies.

\section{Recent Discoveries and Further Applications for TK1}

In the last decade, immunological advances have allowed tumor biomarkers like TK1 to garner marked attention as a diagnostic, prognostic, and therapeutic targets in the clinical setting. Specifically, TK1 expression has been demonstrated to increase in lung, breast, and colon tumor cell lines ${ }^{1}$ indicating that patients presenting any of the aforementioned cancers as potential candidates for TK1-targeted immunotherapy. In an extensive review, Bitter et al. ${ }^{1}$ references a variety of studies that identify TK1 as a potential biomarker found in lung adenocarcinoma ${ }^{34}$, thyroid carcinoma ${ }^{20}$, as well as associated with a variety of other tumor-progression mechanisms ${ }^{35}$. An additional study done by Miran et al. identifies TK1 as a key enzyme in nucleotide synthesis and proliferation of malignant melanoma. Utilizing TK1 as a target both in vitro and in vivo, this preclinical study suggests TK1 as a potential target for "highly efficient and selective" radioactive clinical treatment and possibilities for TK1 targeting in melanoma patients $^{36}$.

Monoclonal antibodies primed to TK1 are utilized as both diagnostic and therapeutic agents for various cancers, including malignant skin cell carcinoma. While approved chemotherapeutic agents have slow response rates with metastatic melanoma, monoclonal antibodies against traditional antigens for the disease induce a notably faster durable response rate $^{37}$. This can be attributed to high target specificity and the accompanying immunotherapeutic effect depending on the antigen of choice. Velazquez et al. developed and evaluated a set of monoclonal antibodies against TK1. Binding of the antibodies was confirmed by Western blot in recombinant protein, cancer serum, and cell lysate, suggesting good affinity $^{38}$. These results combined with the potential efficacy associated with monoclonal antibody treatment in melanoma patients nominate TK1 as an additional target enzyme utilized in treatment.

Chimeric antigen receptor (CAR) technology represents a novel immunotherapeutic class consisting of cloned leukocytes obtained from a diseased host for which the therapy is intended. CAR T-cell therapy is most effective when utilized in presentation of B-cell Leukemia and Lymphoma, as these require systemic attention for remission to occur ${ }^{39}$. Similarly, the diffuse nature of disease in patients presenting with metastatic melanoma suggests clinical potency in utilizing CAR T-cells with anti-TK1 receptors. A limitation associated with CAR T-therapy is nullification by the microenvironment typically associated with tumor. Particularly new development and discussion of CAR-Macrophage therapy, however, shows promise. Macrophages are known to aggregate around and interact with the tumor microenvironment ${ }^{40}$, facilitating a more focused immunotherapeutic reaction against the desired solid tumor while mitigating patient harm, indicating compatibility as a vehicle in TK1-associated immunotherapy.

\section{Conclusions}

As technological advancements are made, there is increased opportunity for further discovery and application of clinically relevant biomarkers in the diagnosing of cancers and progression of effective treatment. When upregulated, the salvage pathway enzyme Thymidine Kinase 1 is shown to be in direct correlation with cancer stage. In this manner, TK1 provides a platform as a biomarker for the tracking of cancer progression and possible immunotargeting during treatment. Substantial research already implementing TK1 as a biomarker and immune target for various cancers has 
produced positive results. Further research of biomarkers such as TK1 has potential to improve the diagnosis and prognosis of cancers.

\section{Conflict of Interest}

There are no conflicts of interest to report.

\section{References}

1. Bitter EE, Townsend MH, Erickson R, et al. Thymidine kinase 1 through the ages: a comprehensive review. Cell Biosci 2020 101.2020 10(1): 1-16. doi:10.1186/S13578-020-00493-1

2. Bradshaw HD. Molecular cloning and cell cycle-specific regulation of a functional human thymidine kinase gene. Proc Natl Acad Sci. 1983; 80(18): 5588-5591. doi:10.1073/PNAS.80.18.5588

3. Topolcan $\mathrm{O}$, Jr LH. The role of thymidine kinase in cancer diseases. http://dx.doi.org/101517/1753005922129. 2008; 2(2): 129-141. doi:10.1517/17530059.2.2.129

4. Jagarlamudi KK, Shaw M. Thymidine kinase 1 as a tumor biomarker: technical advances offer new potential to an old biomarker. $h$ ttps://doi. org/102217/bmm-2018-0157. 2018; 12(9): 1035-1048. doi:10.2217/ BMM-2018-0157

5. O’Neill KL, Buckwalter MR, Murray BK. Expert Review of Molecular Diagnostics Thymidine kinase: diagnostic and prognostic potential. Published online 2014. doi:10.1586/14737159.1.4.428

6. Wu BJ, Li WP, Qian C, et al. Increased serum level of thymidine kinase 1 correlates with metastatic site in patients with malignant melanoma. Tumor Biol. 2013; 34(2): 643-648. doi:10.1007/S13277-012-0591-0

7. LJ Bello. Regulation of thymidine kinase synthesis in human cells. Exp Cell Res. 1974; 89(2): 263-274. doi:10.1016/0014-4827(74)90790-3

8. Cautain B, Hill R, de Pedro $\mathrm{N}$, et al. Components and regulation of nuclear transport processes. FEBS J. 2015, 282, 445-462, doi:10.1111/FEBS.13163.

9. Chen Y-L, Eriksson S, Chang Z-F. Regulation and Functional Contribution of Thymidine Kinase 1 in Repair of DNA Damage. J Biol Chem. 2010; 285(35): 27327. doi:10.1074/JBC.M110.137042

10. Wenzel ES, Singh ATK. Cell-cycle Checkpoints and Aneuploidy on the Path to Cancer. In Vivo (Brooklyn). 2018, 32, 1, doi:10.21873/INVIVO.11197

11. Adler R, McAuslan BR. Expression of thymidine kinase variants is a function of the replicative state of cells. Cell. 1974; 2(2): 113-117. doi:10.1016/0092-8674(74)90100-7

12. Zhu $\mathrm{X}$, Shi $\mathrm{C}$, Peng $\mathrm{Y}$, et al. Thymidine kinase 1 silencing retards proliferative activity of pancreatic cancer cell via E2F1-TK1-P21 axis. Cell Prolif. 2018; 51(3): 51. doi:10.1111/CPR.12428

13. Kim YK, Lee AS. Identification of a 70-base-pair cell cycle regulatory unit within the promoter of the human thymidine kinase gene and its interaction with cellular factors. Mol. Cell. Biol. 1991, 11, 2296, doi:10.1128/MCB.11.4.2296

14. Munch-Petersen B, Cloos L, Jensen HK, et al. Human thymidine kinase 1. Regulation in normal and malignant cells. Adv. Enzyme Regul. 1995, 35, 69-89, doi:10.1016/0065-2571(94)00014-T.

15. TR Breitman. The feedback inhibition of thymidine kinase Biochim Biophys Acta. 1963; 67(C): 153-155. doi:10.1016/00063002(63)91807-9

16. Weber G, Lea MA. The molecular correlation concept of neoplasia Adv Enzyme Regul. 1966; 4(C): 115-145. doi:10.1016/00652571(66)90011-2

17. Alegre MM, Weyant MJ, Bennet DT, et al. Serum Detection of Thymidine Kinase 1 as a Means of Early Detection of Lung Cancer. Anticancer Res. 2014; 34 (5).
18. Matikas A, Wang K, Lagoudaki E, et al. Prognostic role of serum thymidine kinase 1 kinetics during neoadjuvant chemotherapy for early breast cancer. ESMO Open. 2021; 6(2): 100076. doi:10.1016/J. ESMOOP.2021.100076

19. Larsson A-M, Bendahl P-O, Aaltonen K, et al. Serial evaluation of serum thymidine kinase activity is prognostic in women with newly diagnosed metastatic breast cancer. Sci Reports 2020 101. 2020; 10(1): 1-11. doi:10.1038/s41598-020-61416-1

20. Liu C, Wang J, Zhao L, et al. Knockdown of Thymidine Kinase 1 Suppresses Cell Proliferation, Invasion, Migration, and EpithelialMesenchymal Transition in Thyroid Carcinoma Cells. Front Oncol. 2020; 0: 1475. doi:10.3389/FONC.2019.01475

21. Weagel EG, Meng W, Townsend MH, et al. Biomarker analysis and clinical relevance of TK1 on the cell membrane of Burkitt's lymphoma and acute lymphoblastic leukemia. Onco Targets Ther. 2017; 10: 4355-4367. doi:10.2147/OTT.S141239

22. Kasprzak A. The Role of Tumor Microenvironment Cells in Colorectal Cancer (CRC) Cachexia. Int J Mol Sci 2021, Vol 22, Page 1565. 2021; 22(4): 1565. doi:10.3390/IJMS22041565

23. Wang J, Wang J, Gu Q et al. TGF $\beta 1$ : An Indicator for Tumor Immune Microenvironment of Colon Cancer From a Comprehensive Analysis of TCGA. Front Genet. 2021; 12: 612011. doi:10.3389/ FGENE.2021.612011

24. Liu B, Yan L, Zhou M. Target selection of CAR T cell therapyin accordance with the TME for solid tumors. Am J Cancer Res. 2019;9(2):228. Accessed October 10, 2021. /pmc/articles/PMC6405971/

25. Rao D, Verburg F, Renner K, et al. Metabolic profiles of regulatory T cells in the tumour microenvironment. Cancer Immunol Immunother 2021 709. 2021; 70(9): 2417-2427. doi:10.1007/S00262-02102881-Z

26. Nachef M, Ali AK, Almutairi SM, et al. Targeting SLC1A5 and SLC3A2/ SLC7A5 as a Potential Strategy to Strengthen Anti-Tumor Immunity in the Tumor Microenvironment. Front Immunol. 2021; 12: 1. doi:10.3389/FIMMU.2021.624324

27. Heiden VM. Cell Programmed Nutrient Partitioning in the Tumor Microenvironment. doi:10.1101/2020.08.10.238428

28. Hamada T, Nowak JA, Milner DA, et al. Integration of microbiology, molecular pathology, and epidemiology: a new paradigm to explore the pathogenesis of microbiome-driven neoplasms. J Pathol. 2019; 247(5): 615. doi:10.1002/PATH.5236

29. Cani PD. Human gut microbiome: hopes, threats and promises. Gut. 2018; 67(9): 1716-1725. doi:10.1136/GUTJNL-2018-316723

30. Khan AA, Shrivastava A, Khurshid M. Normal to cancer microbiome transformation and its implication in cancer diagnosis. Biochim Biophys Acta - Rev Cancer. 2012; 1826(2): 331-337. doi:10.1016/J. BBCAN.2012.05.005

31. Whitt DD, Savage DC. Influence of indigenous microbiota on activities of alkaline phosphatase, phosphodiesterase I, and thymidine kinase in mouse enterocytes. Appl Environ Microbiol. 1988; 54(10): 24052410. doi:10.1128/AEM.54.10.2405-2410.1988

32. Hamada T, Keum NN, Nishihara R, et al. Molecular pathological epidemiology: new developing frontiers of big data science to study etiologies and pathogenesis. J Gastroenterol 2016 523. 2016; 52(3): 265-275. doi:10.1007/S00535-016-1272-3

33. Ogino $\mathrm{S}$, Nowak JA, Hamada $\mathrm{T}$, et al. Insights into Pathogenic Interactions Among Environment, Host, and Tumor at the Crossroads of Molecular Pathology and Epidemiology. doi:10.1146/ANNUREVPATHMECHDIS-012418-012818

34. Tilli TM, Carels N, Tuszynski JA, et al. Validation of a network-based strategy for the optimization of combinatorial target selection in 
breast cancer therapy: siRNA knockdown of network targets in MDA-MB-231 cells as an in vitro model for inhibition of tumor development. Oncotarget. 2016; 7(39): 63189-63203. doi:10.18632/ ONCOTARGET.11055

35. Malvi P, Janostiak R, Nagarajan A, et al. Loss of Thymidine Kinase 1 Inhibits Lung Cancer Growth and Metastatic Attributes by Reducing GDF15 Expression. PLoS Genetics 2019, 15, doi:10.1371/journal. pgen.1008439.

36. Miran T, Vogg ATJ, Moussaoui L El, et al. Dual addressing of thymidine synthesis pathways for effective targeting of proliferating melanoma. Cancer Med. 2017; 6(7): 1639-1651. doi:10.1002/CAM4.1113
37. Bhandaru M, Rotte A. Monoclonal Antibodies for the Treatment of Melanoma: Present and Future Strategies. Methods in Molecular Biology 2019, 1904, 83-108, doi:10.1007/978-1-4939-8958-4_4.

38. Velazquez EJ, Brindley TD, Shrestha G, et al. Novel monoclonal antibodies against thymidine kinase 1 and their potential use for the immunotargeting of lung, breast and colon cancer cells. Cancer Cell Int 2020 201. 2020; 20(1): 1-20. doi:10.1186/S12935-020-01198-8

39. Sterner RC, Sterner RM. CAR-T cell therapy: current limitations and potential strategies. Blood Cancer J 2021 114. 2021; 11(4): 1-11. doi:10.1038/s41408-021-00459-7

40. Chen Y, Yu Z, Tan X, et al. CAR-Macrophage: A New Immunotherapy Candidate against Solid Tumors. Biomedicine and Pharmacotherapy 2021, 139. doi:10.1016/J.BIOPHA.2021.111605 